\title{
Identification a ceRNA (XIST/miR-10a- 5p/SERPINE1) Axis as a Prognostic Biomarker in Kidney Renal Clear Cell Carcinoma.
}

Rui-ji Liu

Southeast University Zhongda Hospital

\section{Zhi-Peng Xu}

Southeast University Zhongda Hospital

\section{Shuying Li}

Sichuan Cancer Hospital and Research Institute: Sichuan Cancer Hospital and Institute

Jun-Jie Yu

Southeast University Zhongda Hospital

\section{Bin Xu}

Southeast University Zhongda Hospital

Ming Chen ( $\square$ 230208925@seu.edu.cn )

Affiliated Zhongda hospital of Southeast University https://orcid.org/0000-0002-3572-6886

\section{Primary research}

Keywords: ceRNA, DNA methylation, immune microenvironment, kidney renal clear cell carcinoma, prognosis

Posted Date: October 1st, 2021

DOl: https://doi.org/10.21203/rs.3.rs-717299/v1

License: (9) This work is licensed under a Creative Commons Attribution 4.0 International License. Read Full License 


\section{Abstract}

Background: Kidney cancer is one of the most common malignancies, of which the most aggressive subtype was kidney renal clear cell carcinoma (KIRC), accounting for $80 \%$ of them. A growing number of studies point to the involvement of competitive endogenous RNAs in tumor development. However, the role of ceRNA network involved in KIRC remains unclear. Thus, the aim of this study was to investigate the BAP1-associated prognostic ceRNA in KIRC.

Methods: We downloaded the RNAseq data from TCGA along with the relevant clinical data. We screened the differentially expressed IncRNAs, miRNAs, mRNAs according to the expression of BAP1 and established a ceRNA network.

Results: After comprehensive bioinformatics analysis, we identified the XIST-miR-10a-5p-SERPINE1 ceRNA axis. Next, we confirmed the prognostic role of miR-10a-5p/SERPINE1 in KIRC using survival analysis and Cox regression analysis. To investigate the abnormally high expression of SERPINE1, we performed methylation analysis of SERPINE1 and concluded that the methylation level of SERPINE1 in KIRC was significantly lower than that in normal tissues. Furthermore, to study the role of SERPINE1 in the immune microenvironment in KIRC, we performed immune cell infiltration analysis and found that SERPINE1 expression was positively correlated with the level of multiple immune cell infiltration (CD 4+ T cell, CD 8+ T cell, macrophages, dendritic cells, neutrophils).

Conclusion: We constructed a ceRNA (XIST/has-miR-10a-5p/SERPINE1) that can be used as prognostic biomarker of KIRC. Furthermore, we found that miR-10a-5p/SERPINE1 were significantly associated with clinical features and were independent prognostic factors of KIRC.

\section{Introduction}

Kidney cancer is a common malignancy, with about 430,000 new cases worldwide in 2020, of which approximately 170,000 will die from the disease [1]. The pathology of kidney renal clear cell carcinoma (KIRC) is characterized by a "clear cytoplasm" due to its ability to accumulate glycogen and lipids in the cytoplasm. It accounts for up to $80 \%$ of renal cell carcinomas and is also the most aggressive subtype. In the early stages, there is an asymptomatic phase, and it usually diagnosed inadvertently by imaging. In the advanced stage of the disease, chemotherapy and immunotherapy bring limited benefit, treatment will be tricky $[2,3]$.

Numerous studies have shown that transcription is present in about $80 \%$ of the human genome, while protein-coding genes account for only $2 \%$, which suggests that the majority of RNAs are non-coding genes [4]. Increasing evidence indicated that the non-coding transcriptome is often dysregulated in cancer and plays an important role in determining the pathogenesis of the disease [5, 6]. Long noncoding RNAs (IncRNA), longer than $200 \mathrm{nt}$, are non-protein-coding RNAs that have been reported to be involved in the development of multiple tumors, including in KIRC, and specific IncRNAs are involved in tumor migration, invasion, recurrence, metastasis, and are associated with poor prognosis [7, 8]. MicroRNA (miRNA) is a 
single-stranded non-coding RNA about 19-25nt long, which can bind to the 3 ' untranslated region of mRNA and regulate the expression of target genes [9]. Non-coding RNAs (including long non-coding RNAs, circular RNAs) act as competitive endogenous RNAs (ceRNA) that can competitively bind miRNAs (a post-transcriptional regulator) to communicate with each other and co-regulation in gene expression [10-12]. In KIRC, the topic of ceRNA regulation network is a hotpot. For example, Liu et al. had found that IncRNA SNHG14 could act as a ceRNA, and mediates migration and invasion of renal clear cell carcinoma by upregulating mRNA N-WASP expression through sponge miR-203 [13]; IncRNA CDKN2BAS1/miR-141/cyclin D ceRNA axis involved in regulation the progression and metastasis of renal cell carcinoma (RCC) [14].

The ubiquitin carboxy-terminal hydrolase BAP1 is a subfamily member of deubiquitinating enzymes, and serve as a tumor suppressor $[15,16]$. An increasing number of studies have reported the presence of mutated BAP1 in numerous of human malignancies, especially in KIRC, hepatocellular carcinoma, mesothelioma [17]. The majority of chromosomes have a 3p deletion, which is an initial factor of nonhereditary KIRC [18]. While the mutated frequency of BAP1, which resides on 3p reached as high as $20 \%$ in KIRC $[19,20]$. Previous studies have shown a role of BAP1 in regulating several pathways, such as upregulating the expression of enhancer of zeste homolog 2 (EZH2); interaction with O-GIcNAc transferase (OGT) and host cell factor-1 (HCT-1) [21-23].

However, few studies have reported on the BAP1-related ceRNA network. In this study, we aimed to construct a BAP1-associated prognostic ceRNA in KIRC by comprehensive bioinformatics analysis. Finally, we identified the XIST-miR-10a-5p-SERPINE1 as prognostic ceRNA, and further conducted methylation analysis and immune cell infiltration analysis of SERPINE1 to investigate the abnormal upregulation of SERPINE1 expression and the relation with immune microenvironment.

\section{Methods And Materials}

\subsection{Data collection and processing}

RNAseq (IncRNA, mRNA and miRNA) and relevant clinical data for kidney renal clear cell carcinoma (KIRC) were obtained from TCGA database (https://portal.gdc.cancer.gov/), and were sorted into normalized transcripts per million reads (TPM) format for subsequent analysis.

\subsection{Identification of different expressed IncRNAs, miRNAs, and mRNAs}

According to the median expression of BAP1, patients were divided into BAP1 $1^{\text {high }}$ and BAP1 $1^{\text {low }}$ group. When performing differential expressing analysis, the cutoff value of DElncRNA was set at $\mid \log _{2} \mathrm{FCl}>0.5$ and $P$. adj $<0.05$; DEmiRNA with cutoff value of $\| \log _{2} F C \mid>0.7$ and $P$. adj $<0.05$, DEmRNA with cutoff value of $\| \log _{2} \mathrm{FC} \mid>0.5$ and $P$. adj $<0.05$, respectively.

\subsection{Construction of ceRNA networks}


We downloaded the high conserved microRNA family file from miRcode database (http://mircode.org/), and based on the obtained DElncRNAs to find potentially the miRNAs targeting these DElncRNAs, and take intersections with our obtained DEmiRNAs. Then, these selected miRNAs were put into miRBD database (http://mirdb.org/) [24], and Targetscan database (http://www.targetscan.org/vert_72/) [25] to explore targeting mRNAs and take intersections with obtained DEmRNAs. Finally, the screened DElncRNAs, DEmiRNAs, and DEmRNAs were put into Cytoscape (version 3.6.1) for ceRNA network, and plug-in "cytoHubba" was utilized for hub genes network construction [26, 27].

\subsection{Functional annotation}

To investigate the functional annotation implicated with DEmRNAs, we performed gene ontology (GO) annotation and Kyoto Encyclopedia of Genes and Genomes (KEGG) pathway analysis through the Metascape website (https://metascape.org/) [28].

\subsection{Expression of hub genes and survival analysis}

We compared the expression of the hub genes in tumor and normal tissues based on KIRC dataset with the Wilcoxon rank-sum test $(P<0.05)$. Overall survival (OS) analysis for the expression of hub genes between high- and low expression group were performed, and log-rank test $P<0.05$ was considering of statistical significance.

\subsection{Clinical relevance of the XIST/has-miR-10a- 5p/SERPINE1 axis in KIRC patients}

To explore the clinical relevance of this ceRNAs axis, we evaluated the expression level of XIST, has-miR10a-5p and SERPINE1 with different clinical characteristics, and Bonferroni method was used for correcting the results of multiple hypothesis testing (Dunn's test, $P$. adj < 0.05 ). Moreover, univariate and multivariate Cox regression analysis were conducted to investigated the prognostic significance of clinical features.

\subsection{DNA methylation analysis of SERPINE1}

To investigate the DNA methylation level of SERPINE1, we performed co-expression analysis of SEPRINE1 and three DNA methyltransferases (DNMT1, DNMT3A, DNMT3B). Then, we performed methylation analysis of SERPINE1 between KIRC and normal tissues through online database DiseaseMeth version 2.0 (http://bio-bigdata.hrbmu.edu.cn/diseasemeth/). Moreover, we utilized MEXPRESS (https://mexpress.be/) to further determine the relationship between SERPINE1 expression and DNA methylation status [29].

\subsection{Correlation between immune infiltration and expression of SERPINE1 in KIRC}

We utilized TIMER (https://cistrome.shinyapps.io/timer/) for comprehensive analysis of the relationship between the expression of SERPINE1 and tumor-infiltrating immune cells level, which including neutrophils, macrophages, and dendritic cells, B cells, CD 4 + T cells, and CD8 + T cells [30]. 


\subsection{Software and versions}

R software (x64, version 4.0.3) was used for statistical calculations and graphs (https://www.rproject.org/).

\section{Results}

\subsection{BAP1 acts as a tumor suppressor in KIRC}

A flow diagram of ceRNA construction and analysis is shown in Figure 1. We conducted a pan-cancer analysis to evaluate the RNA expression level of BAP1 using data from UCSC XENA (https://xenabrowser.net/datapages/), and protein expression level through Human Protein Atlas (HPA) database (https://www.proteinatlas.org/). We found that the RNA expression level of BAP1 was significantly downregulated in KIRC (Fig. 2A), and interestingly BAP1 protein level was the lowest in renal cancer (Fig S1). Immunohistochemistry (IHC) staining obtained from HPA also validated the downregulation of BAP1 in tumor tissue (Fig. 2B).

To investigate the relationship between BAP1 and the prognosis of KIRC, we used Kaplan-Meier survival curves to compared the survival of high- and low-BAP1 expression group. Our result indicated that low expression of BAP1 was associated with poor OS in KIRC (Fig. 2C).

Moreover, we utilized cBioPortal (http://www.cbioportal.org/) to explore the potential mechanisms underlying the abnormally lower expression of BAP1 in KIRC [31]. Figure 2D showed the genetic alteration rate of BAP1 is $13 \%$. Gene deletions (deep deletion and shallow deletion) account for more than half of the copy number alterations in KIRC samples (Fig. 2E). In addition, the mRNA expression level of BAP1 is found to be positively correlated with the copy number value (Fig. 2F).

As listed above, BAP1 is down-regulated in KIRC, and deletions of copy number may be one of the potential mechanisms underlying the lower expression level of BAP1 in KIRC.

\subsection{Identification of different expressed IncRNAs, miRNAs, and mRNAs}

We aimed to construct a prognostic-related ceRNA network based on the expression of BAP1. Therefore, we performed differentially expressed genes (DEGs) analysis comparing BAP $1^{\text {high }}$ and BAP $1^{\text {low }}$ group in KIRC samples. Finally, a total of 3425 DElncRNA, 84 DEmiRNA, and 2753 DEmRNA were screened according to the cut-off value (Fig. 3).

\subsection{Construction of IncRNA-miRNA-mRNAs networks}


After taking intersection of the predicted miRNAs with the obtained DEmiRNAs, four miRNAs were selected. Then, we used miRBD database and Targetscan databases to identify the downstream targeting mRNAs of the four predicted miRNAs. Similarly, by taking intersections with DEmRNAs, 387 predicted mRNAs were identified. Finally, 4 IncRNAs, 4 miRNAs, and 387 mRNAs were selected.

Then, we put these genes into Cytoscape (version 3.6.1) for ceRNA network construction (Fig. 4A), and plug-in "cytoHubba" was utilized for hub genes network construction. Finally, IncRNAs (XIST, HELLPAR, PURPL), miRNAs (miR-10a-5p, miR-508-3p, miR-135a-5p), and mRNAs (IRS1, SERPINE1, KCAN1, TRIM2, RORB, SIX4) were identified (Fig. 4B).

We conducted functional enrichment analysis of DEmRNAs to investigate the potential function of these ceRNA networks through Metascape. The result demonstrated that these DEmRNAs were involved in mesenchyme development, transmembrane receptor protein tyrosine kinase signaling pathway, regulation of cell adhesion (Fig. 4C).

\subsection{Construction of prognostic-related ceRNA in KIRC}

To construct a prognostic-related ceRNA, we first analyzed the expression of hub genes comparing tumor and normal tissue, and the results showed that differences were significant in all hub genes (Fig. 5). Then, survival analyses were performed to select prognostic-related genes as shown in Figure 6. In total, two DElncRNAs (XIST, HELLPAR), one DEmiRNA (miR-10a-5p), and four DEmRNAs (SERPINE1, TRIM2, RORB, SIX4) were found to be prognostic-related genes.

We utilized IncLocator (www.csbio.sjtu.edu.cn/bioinf/IncLocator/) to determine the subcellular localization of two DElncRNAs, and found that XIST was mainly distributed in cytoplasm (Fig. 7A), which indicated that XIST may act as a ceRNA in improving the expression of SERPINE1 through sponge of miR-10a-5p. Finally, a prognostic-related XIST/miR-10a-5p/SERPINE1 ceRNA network was constructed (Fig. 7B). In addition, the target sites of XIST and SERPINE1 to miR-10a-5p were predicted by Targetscan (Fig. 7C).

\subsection{Clinical relevance of the XIST/has-miR-10a- 5p/SERPINE1 axis in KIRC patients}

We evaluated the expression level of XIST, has-miR-10a-5p and SERPINE1 with different clinical factors (T stage, N stage, M stage, pTNM stage, tumor grade, gender, and age) to determine the clinical relevance of this ceRNA axis. The results showed that the expression of XIST was correlated with tumor grade, and sex $(P<0.05$, Fig. S2A). Lower expression level of has-miR-10a-5p was found to associated with higher $T$, $M$ stages, pTNM stages, tumor grade and gender $(P<0.05$, Fig. S2B). In addition to age and M stage, the expression level of SERPINE1 was strongly correlated with T stage, $N$ stage, pTNM stage, tumor grade 
and gender $(P<0.05$, Fig. S2C). These findings are generally consistent with the results of survival analysis.

Moreover, we performed univariate and multivariate Cox regression model analysis and ROC (receiver operating characteristic curve) analysis to determine the OS-related prognostic significance of this ceRNA axis. In multivariate Cox regression analysis, SERPINE1 (HR=1.456, $P=0.015)$ and has-miR-10a-5p (HR=0.681, $P=0.014$ ) were found to be independent prognostic factor in KIRC, while XIST was not (Table. S1-3). The AUC (area under curves) of ROC analysis suggested a good prognostic performance of SERPINE1 (AUC=0.789) and has-miR-10a-5p (AUC=0.892). These results were showed in Fig. S3.

In addition, we performed a pan-cancer analysis of SERPINE1 mRNA expression and found that SERPINE1 was highly expressed in kidney cancer (Fig. S4A). Immunohistochemical analysis revealed that SERPINE1 was located in the cytoplasmic/membranous and was only detected in the renal tubules (Fig. S4B).

\subsection{DNA methylation analysis of SERPINE1}

To elucidate the mechanism of the abnormally high expression of SERPINE1 in KIRC, we performed a series of methylation analyses of SERPINE1. Firstly, the results of co-expression analysis suggested that the expression of SERPINE1 was positively correlated with the expression levels of the DNMT1, DNMT3A and DNMT3B $(P<0.05$, Fig. 8A). Secondly, we obtained the same result with DiseaseMeth version 2.0, that the methylation level of SERPINE1 in normal tissues were much higher than in $\operatorname{KIRC}(P<0.001$, Fig. $8 \mathrm{~B})$. Thirdly, we found that the methylation level of SERPINE1 correlated with OS. Moreover, we also identified 12 methylation sites on DNA sequences that were negatively correlated with SERPINE1 expression (Fig. 8C).

\subsection{Immune infiltration analysis of SERPINE1 in KIRC}

To further investigate the relationship between the expression level of SERPINE1 and the immune microenvironment in KIRC, we performed an immune infiltration analysis with TIMER. The "SCNA" module analysis indicated that the immune infiltration level of $C D 4+T$ cell was associated with altered copy numbers of SERPINE1 (Fig. 9A). Moreover, the "Gene" module analysis showed that the expression level of SERPINE1 was positively related with immune infiltration level of CD 4+ T cell, CD 8+ T cell, macrophages, dendritic cells, neutrophils (Fig. 9B).

\section{Discussion}

$\mathrm{KIRC}$ is the most common subtype of renal cell carcinoma with a high lethality rate worldwide [1]. Due to the lack of clinical symptoms in the early stages, the best time for surgical treatment is missed in about $30-50 \%$ of cases at the time of diagnosis, and the 5 -year survival rate of KIRC is less than $20 \%$ when it 
comes to advanced stages [32]. BAP1 is a classical tumor suppressor, involved in several biological processes such as DNA damage repair, cell cycle regulation, programmed cell death, immune microenvironment, metabolic regulation [33,34]. BAP1 is located on the 3p of chromosome, and mutations are seen in a variety of tumors, especially KIRC. While ceRNAs have been reported to be associated with recurrence, invasion, and metastasis of a variety of tumors, the BAP1-acossiated ceRNA network has been little studied. Thus, in this study, we conducted a comprehensive bioinformatics analysis to explore prognostic ceRNA network in KIRC.

In this study, a BAP1-associated ceRNA network was constructed, which included 4 IncRNAs, 4 miRNAs, and 387 mRNAs. We then performed a functional analysis of the differentially expressed mRNAs. The results showed that these DEmRNAs were mainly enriched in mesenchyme development, transmembrane receptor protein tyrosine kinase signaling pathway, regulation of cell adhesion. Receptor tyrosine kinases (RTK) are a family of transmembrane receptors where phosphorylation/activation of the receptor determines the downstream molecule signaling and biological functions [35]. Although many TK-related genes have been shown to be significantly upregulated in KIRC, the mechanisms of RTK in KIRC tumorigenesis have not been fully investigated [36]. Chen et al. found that knockdown of BAP1 increased the expression of TK-related genes; in addition, the expression of BAP1 was closely associated with epithelial mesenchymal transition (EMT) and cell adhesion [37]. Next, the plug-in "cytoHubba" was used to build the hub gene network, which including three IncRNAs (XIST, HELLPAR, PURPL), three miRNAs (miR-10a-5p, miR-508-3p, miR-135a-5p), and six mRNAs (IRS1, SERPINE1, KCAN1, TRIM2, RORB, SIX4). Furthermore, we performed gene expression analyses and survival analyses of these hub genes. Finally, two DEIncRNA (XIST, HELLPAR), one DEmiRNA (miR-10a-5p), and four DEmRNA (SERPINE1, TRIM2, RORB, SIX4) were confirmed to be prognostic-related ceRNAs. Given that the biological processes of ceRNAs are mainly localized in the cytoplasm, we conducted subcellular localization analyses of these two IncRNAs. The results showed that IncRNA XIST was mainly distributed in the cytoplasm. Finally, a prognostic-related XIST/miR-10a-5p/SERPINE1 ceRNA triple regulation axis was confirmed.

Previous studies had reported that XIST is highly expressed in several tumors and is involved in the regulation of tumor cell proliferation, migration and invasion as ceRNA [38-40]. Meanwhile, up-regulated miR-10a-5p inhibited proliferation of cancer cell, and promoted cell apoptosis [41-43]. Serine protease inhibitor family E member 1 (SERPINE1), encoding plasminogen activator inhibitor 1 (PAI-1), is upregulated in different types of tumors, and is involved in regulating the biological processes of tumor cells through various pathways like: NF-KB signaling, TGF- $\beta$ signaling, TNFa signaling, Notch1 Signaling, EMT [44-48].

In the present study, the expression of XIST and SERPINE1 was significantly higher in KIRC than in normal tissues. High expression of XIST and SERPINE1 in KIRC was associated with poor prognosis by survival analysis. In contrast, hsa-miR-10a-5p expression was higher in normal tissues than in KIRC, and low expression of hsa-miR-10a-5p was associated with poor prognosis. Furthermore, we analyzed the correlation of XIST/miR-10a-5p/SERPINE1 axis with clinical characteristics, and concluded that the expression of XIST did not differ in different T stage, N stage, M stage, PTNM stage and age. However, 
the expression of hsa-miR-10a-5p and SERPINE1 were significantly different with in different clinical characteristics. In addition, we performed a multivariate Cox regression model analysis, and found that high expression of SERPINE1 was an independent risk factor for poor OS in KIRC; high expression of hsamiR-10a-5p was a protective factor for OS. ROC analysis also suggested that hsa-miR-10a-5p and SERPINE1 had good predictive value in KIRC (AUC>0.75). The above results support the predictive efficacy of this ceRNA.

DNA methylation is defined as the addition of a methyl group to a cytosine base to form 5-methylcytosine [49], which serves as regulator in gene expression by recruiting proteins that repress gene expression and by blocking transcription factors from binding to DNA [50]. DNA methylation is abnormal in all types of tumors. Decreased overall methylation levels and increased methylation of $\mathrm{CpG}$ islands in the promoter region are important mechanisms of aberrant methylation in tumors [51]. To elucidate the mechanism of the abnormally high expression of SERPINE1 in KIRC, we performed a series of methylation analyses on SERPINE1 using online websites. Three DNA methyltransferases (DNMT1, DNMT3A, DNMT3B) are important for the establishment and maintenance of DNA methylation patterns [52]. Therefore, we used co-expression analysis to analyze the correlation of SERPINE1 with three DNA methyltransferases (DNMT), and the results showed that SERPINE1 was positively correlated with the expression of three DNMTs $(P<0.05)$. In addition, we found that the methylation level of SERPINE1 in KIRC was significantly lower than that of normal tissue, which was consistent with the results of co-expression analysis of DNMTs. Furthermore, we used MEXPRESS to explore the relationship between SERPINE1 expression and genome-wide methylation, and found that 12 methylation sites were significantly negatively correlated with SERPINE1 expression. Therefore, we suggest that the abnormal increase in SERPINE1 expression is associated with SERPINE1 hypomethylation, which may be related to the poor prognosis of KIRC patients.

The tumor immune microenvironment determines the biological behavior of tumor cells, and previous studies have suggested that the level of immune cell infiltration correlates with prognosis of tumor patients [53-55]. Different types of immune cell infiltration have distinct value on predicting the response to immunotherapy. Inhibitors that target the immune system may respond best to T-cell-infiltrated tumors [56]. In the present study, we found that the copy number of SERPINE1 was associated with immune infiltration of CD4+ T cells, and that the expression of SERPINE1 was related to the level of immune infiltration of CD 4+ T cells, CD 8+ T cells, macrophages, dendritic cells, and neutrophils. These findings suggested that XIST/has-miR-10a-5p/SERPINE1 axis may be involved in the regulation of the immune microenvironment of KIRC and may be potential targets for immunotherapy.

Although we established the prognosis-related XIST/has-miR-10a-5p/SERPINE1 ceRNA regulatory network, further experiments are still need to validate the regulation, function, and mechanism of these ceRNAs.

\section{Conclusions}


In conclusion, we constructed a ceRNA (XIST/has-miR-10a-5p/SERPINE1) that can be used as prognostic biomarker of KIRC. Furthermore, we found that miR-10a-5p/SERPINE1 were significantly associated with clinical features and were independent prognostic factors of KIRC, which could help understand the carcinogenesis of KIRC.

\section{Abbreviations}

KIRC, kidney renal clear cell carcinoma; IncRNA, long noncoding RNA; miRNA, microRNA; ceRNA, competitive endogenous RNA; EZH2, enhancer of zeste homolog 2; OGT, O-GIcNAc transferase; HCT-1, host cell factor-1; TCGA, The Cancer Genome Atlas; DElncRNA, differentially expressed IncRNA; DEmiRNA, differentially expressed miRNA; DEmRNA, differentially expressed mRNA; KEGG, Kyoto Encyclopedia of Genes and Genomes; GO, Gene ontology; OS, Overall survival; HPA, Human Protein Atlas; IHC, immunohistochemistry; DEGs, differentially expressed genes; ROC, receiver operating characteristic curve; AUC, area under curves; EMT, epithelial mesenchymal transition; DNMT, DNA methyltransferase.

\section{Declarations}

\section{AUTHOR CONTRIBUTIONS}

RJL conducted study design, data extraction and analysis. RJL, ZPX, JJY and BX wrote the manuscript. $\mathrm{RJL}, \mathrm{BX}$ and $\mathrm{MC}$ reviewed and revised the manuscript. All authors read and approved the final manuscript for publication.

\section{AVAILABILITY OF DATA AND MATERIALS}

The datasets used and/or analyzed during the current study are available from the corresponding author on reasonable request.

\section{CONFLICTS OF INTEREST}

All authors have no conflict of interest.

\section{ACKNOWLEGEMENTS}

We thank all the authors for the support in this study.

\section{ETHICS APPROVAL}

Not applicable. 


\section{FUNDING}

This study was funded by The National Natural Science Foundation of China (No. 81872089, 81370849, 81672551, 81300472, 81070592, 81202268, 81202034); Six talent peaks project in Jiangsu Province, Jiangsu Provincial Medical Innovation Team (CXTDA2017025); Natural Science Foundation of Jiangsu Province (BK20161434, BL2013032, BK20150642 and BK2012336).

\section{ORCID}

Ming Chen: https://orcid.org/0000-0002-3572-6886.

\section{References}

1. Sung HA-O, et al. Global Cancer Statistics 2020: GLOBOCAN Estimates of Incidence and Mortality Worldwide for 36 Cancers in 185 Countries. CA Cancer J Clin. 2021;71(3):(1542-4863 (Electronic)): p. 209-249.

2. Di Lorenzo G, et al. The evolving role of monoclonal antibodies in the treatment of patients with advanced renal cell carcinoma: a systematic review. Expert Opin Biol Ther. 2016;16(11):(1744-7682 (Electronic)): p. 1387-1401.

3. Makhov P, et al. Resistance to Systemic Therapies in Clear Cell Renal Cell Carcinoma: Mechanisms and Management Strategies. Mol Cancer Ther. 2018;17(7):(1538-8514 (Electronic)): p. 1355-1364.

4. Tay Y, Rinn J, Pandolfi PP. The multilayered complexity of ceRNA crosstalk and competition. Nature. 2014;505(7483):(1476-4687 (Electronic)): p. 344 - 52.

5. Mattick JS. Non-coding RNAs: the architects of eukaryotic complexity. EMBO Rep. 2001;2(11): (1469-221X (Print)): p. $986-91$.

6. Mattick JS, Gagen MJ. The evolution of controlled multitasked gene networks: the role of introns and other noncoding RNAs in the development of complex organisms. Mol Biol Evol. 2001;18(9):(07374038 (Print)): p. 1611-30.

7. Hu G, et al., Hypoxia-induced IncHILAR promotes renal cancer cell invasion and metastasis via ceRNA for the miR-613/206/1-1-3p/Jagged-1/Notch/CXCR4 signaling pathway. LID - S15250016(21)00302-6 [pii] LID - 10.1016/j.ymthe.2021.05.020 [doi]. Mol Ther, 2021. S1525-0016(21) (1525-0024 (Electronic)): p. 00302-6.

8. Hong $Q$, et al. LncRNA HOTAIR regulates HIF-1 $\mathrm{a} / \mathrm{AXL}$ signaling through inhibition of miR-217 in renal cell carcinoma. Cell Death Dis. 2017;8(5):(2041-4889 (Electronic)): p. e2772.

9. Fabian MR, Sonenberg N. The mechanics of miRNA-mediated gene silencing: a look under the hood of miRISC. Nat Struct Mol Biol. 2012;9(6):(1545-9985 (Electronic)): p. 1586-93.

10. Cazalla D, Yario JA, Fau - T, Steitz, Steitz JA. Down-regulation of a host microRNA by a Herpesvirus saimiri noncoding RNA. Science. 2010;328(5985):(1095-9203 (Electronic)): p. 1563-6. 
11. Franco-Zorrilla JM, et al. Target mimicry provides a new mechanism for regulation of microRNA activity. Nat Genet. 2007;39(8):(1061-4036 (Print)): p. 1033-7.

12. Poliseno $L$, et al. A coding-independent function of gene and pseudogene mRNAs regulates tumour biology. Nature. 2010;465(7301):(1476-4687 (Electronic)): p. 1033-8.

13. Liu G, et al. SP1-induced up-regulation of IncRNA SNHG14 as a ceRNA promotes migration and invasion of clear cell renal cell carcinoma by regulating N-WASP. Am J Cancer Res. 2017;7(12): (2156-6976 (Print)): p. 2515-2525.

14. Dasgupta PA-O, et al. LncRNA CDKN2B-AS1/miR-141/cyclin D network regulates tumor progression and metastasis of renal cell carcinoma. Cell Death Dis. 2020;11(8):(2041-4889 (Electronic)): p. 660.

15. Carbone M, et al. Biological Mechanisms and Clinical Significance of BAP1 Mutations in Human Cancer. Cancer Discov. 2020;10(8):(2159-8290 (Electronic)): p. 1103-1120.

16. Carbone M, et al. BAP1 and cancer. Nat Rev Cancer. 2013;13(3):(1474-1768 (Electronic)): p. 153-9.

17. Masclef $L A-O$, et al. Roles and mechanisms of BAP1 deubiquitinase in tumor suppression. Cell Death Differ. 2021;28(2):(1476-5403 (Electronic)): p. 606-625.

18. Jonasch, EA.-O., CL, Walker, Rathmell WA-O. Clear cell renal cell carcinoma ontogeny and mechanisms of lethality. Nat Rev Nephrol. 2021;17(4):(1759-507X (Electronic)): p. 245-261.

19. Kapur P, et al. Effects on survival of BAP1 and PBRM1 mutations in sporadic clear-cell renal-cell carcinoma: a retrospective analysis with independent validation. Lancet Oncol. 2013;14(2):(14745488 (Electronic)): p. 159-167.

20. Peña-Llopis S, et al. BAP1 loss defines a new class of renal cell carcinoma. Nat Genet. 2012;44(7): (1546-1718 (Electronic)): p. 751-9.

21. Dey A, et al. Loss of the tumor suppressor BAP1 causes myeloid transformation. Science. 2012;337(6101):(1095-9203 (Electronic)): p. 1541-6.

22. Sun $\mathrm{C}$, et al. EZH2 Expression is increased in BAP1-mutant renal clear cell carcinoma and is related to poor prognosis. J Cancer. 2018;9(20):(1837-9664 (Print)): p. 3787-3796.

23. Machida YJ, et al. The deubiquitinating enzyme BAP1 regulates cell growth via interaction with HCF1. J Biol Chem. 2009;284(49):(1083-351X (Electronic)): p. 34179-88.

24. Chen $Y$, Wang $X$, miRDB: an online database for prediction of functional microRNA targets. Nucleic Acids Res, 2020. 48(D1)(1362-4962 (Electronic)): p. 127-131.

25. Agarwal V, et al., Predicting effective microRNA target sites in mammalian mRNAs. LID 10.7554/eLife.05005 [doi] LID - e05005. Elife, 2015. 4(2050-084X (Electronic)): p. e05005.

26. Chin CF - Chen S-H, et al., cytoHubba: identifying hub objects and sub-networks from complex interactome. BMC Syst Biol, 2014. Suppl 4(1752 - 0509 (Electronic)): p. S11.

27. Shannon $P$, et al. Cytoscape: a software environment for integrated models of biomolecular interaction networks. Genome Res. 2003;13(11):(1088-9051 (Print)): p. 2498 - 504.

28. Zhou Y, et al. Metascape provides a biologist-oriented resource for the analysis of systems-level datasets. Nat Commun. 2019;10(1):(2041-1723 (Electronic)): p. 1523. 
29. Koch A, et al., MEXPRESS update 2019. Nucleic Acids Res, 2019. 47(W1)(1362-4962 (Electronic)): p. W561-W565.

30. Li T, et al. TIMER: A Web Server for Comprehensive Analysis of Tumor-Infiltrating Immune Cells. Cancer Res. 2017;77(21):(1538-7445 (Electronic)): p. e108-e110.

31. Cerami $E$, et al. The cBio cancer genomics portal: an open platform for exploring multidimensional cancer genomics data. Cancer Discov. 2012;2(5):(2159-8290 (Electronic)): p. 401-4.

32. Hsieh JJ, et al. Chromosome 3p Loss-Orchestrated VHL, HIF, and Epigenetic Deregulation in Clear Cell Renal Cell Carcinoma. J Clin Oncol. 2018;36(36):(1527-7755 (Electronic)): p. JC02018792549.

33. Zhang Y, et al. BAP1 links metabolic regulation of ferroptosis to tumour suppression. Nat Cell Biol. 2018;20(10):(1476-4679 (Electronic)): p. 1181-1192.

34. Louie BH, Kurzrock R. BAP1: Not just a BRCA1-associated protein. Cancer Treat Rev. 2020;90(Electronic):1532-967. p. 102091.

35. Schlessinger J. Cell signaling by receptor tyrosine kinases. Cell. 2000;103(2):(0092-8674 (Print)): p. $211-25$.

36. Behbahani TE, et al. Tyrosine kinase expression profile in clear cell renal cell carcinoma. World J Urol. 2012;30(4):(1433-8726 (Electronic)): p. $559-65$.

37. Chen P, et al. Loss of BAP1 Results in Growth Inhibition and Enhances Mesenchymal-Epithelial Transition in Kidney Tumor Cells. Mol Cell Proteomics. 2019;18(7):(1535-9484 (Electronic)): p. $1320-1329$.

38. Liu H, et al. LncRNA XIST/miR-34a axis modulates the cell proliferation and tumor growth of thyroid cancer through MET-PI3K-AKT signaling. J Exp Clin Cancer Res. 2018;37(1):(1756-9966 (Electronic)): p. 279.

39. Jiang $\mathrm{H}$, et al. Knockdown of long non-coding RNA XIST inhibits cell viability and invasion by regulating miR-137/PXN axis in non-small cell lung cancer. Int $\mathrm{J}$ Biol Macromol. 2018;111(Electronic):1879-0003. p. 623-631.

40. Zhao Y, et al. IncRNA-Xist/miR-101-3p/KLF6/C/EBPa axis promotes TAM polarization to regulate cancer cell proliferation and migration. Mol Ther Nucleic Acids. 2020;23(Print):2162-531. p. 536551.

41. Zhang J, et al., S-equol inhibits proliferation and promotes apoptosis of human breast cancer MCF-7 cells via regulating miR-10a-5p and PI3K/AKT pathway. Arch Biochem Biophys, 2019. 672(1096 0384 (Electronic)): p. 108064.

42. Hao SC, et al., hUC-MSCs secreted exosomes inhibit the glioma cell progression through PTENP1/miR-10a-5p/PTEN pathway. Eur Rev Med Pharmacol Sci, 2019. 23(22)(2284 - 0729 (Electronic)): p. 10013-10023.

43. Guo L, et al. RECQL4, Negatively Regulated by miR-10a-5p, Facilitates Cell Proliferation and Invasion via MAFB in Ovarian Cancer. Front Oncol. 2020;10:2234-943X (Print)): p. 524128. 
44. McCann JV, et al. Endothelial miR-30c suppresses tumor growth via inhibition of TGF- $\beta$-induced Serpine1. J Clin Invest. 2019;129(4):(1558-8238 (Electronic)): p. 1654-1670.

45. Wang X, et al., Downregulation of the Ubiquitin-E3 Ligase RNF123 Promotes Upregulation of the NFKB1 Target SerpinE1 in Aggressive Glioblastoma Tumors. LID - 10.3390/cancers12051081 [doi] LID - 1081. Cancers (Basel), 2020. 12(5)(2072-6694 (Print)): p. 1081.

46. Qu X, et al. TNFa-Erk1/2 signaling pathway-regulated SerpinE1 and SerpinB2 are involved in lipopolysaccharide-induced porcine granulosa cell proliferation. Cell Signal. 2020;73(Electronic):1873-3913. p. 109702.

47. Yu XM, et al., Notch1 Signaling Regulates the Aggressiveness of Differentiated Thyroid Cancer and Inhibits SERPINE1 Expression. Clin Cancer Res, 2016. 22(14)(1557-3265 (Electronic)): p. 3582-92.

48. Dittmann $M$, et al. A serpin shapes the extracellular environment to prevent influenza $A$ virus maturation. Cell. 2015;160(4):(1097-4172 (Electronic)): p. 631-643.

49. He XJ, Chen J-K, Fau - T, Zhu, Zhu JK. Regulation and function of DNA methylation in plants and animals. Cell Res. 2011;21(3):(1748-7838 (Electronic)): p. $442-65$.

50. Moore LD, Le G, Fau - T, Fan, Fan G. DNA methylation its basic function Neuropsychopharmacology. 2013;38(1):(1740-634X (Electronic)): p. 23-38.

51. Klutstein M, et al. DNA Methylation in Cancer and Aging. Cancer Res. 2016;76(12):(1538-7445 (Electronic)): p. 3446-50.

52. Jin B, et al. DNMT1 and DNMT3B modulate distinct polycomb-mediated histone modifications in colon cancer. Cancer Res. 2009;69(18):(1538-7445 (Electronic)): p. 7412-21.

53. Pagès $F$, et al. Effector memory $T$ cells, early metastasis, and survival in colorectal cancer. $\mathrm{N}$ Engl $\mathrm{J}$ Med. 2005;353(25):(1533-4406 (Electronic)): p. 2654-66.

54. Galon J, et al. Type, density, and location of immune cells within human colorectal tumors predict clinical outcome. Science. 2006;313(5795):(1095-9203 (Electronic)): p. 960-4.

55. Mlecnik B, et al. Histopathologic-based prognostic factors of colorectal cancers are associated with the state of the local immune reaction. J Clin Oncol. 2011;29(6):(1527-7755 (Electronic)): p. 610-8.

56. Gajewski TF, Schreiber Y-X, Fau - H, Fu, Fu YX. Innate and adaptive immune cells in the tumor microenvironment. Nat Immunol. 2013;14(10):(1529-2916 (Electronic)): p. 1014-22.

\section{Figures}




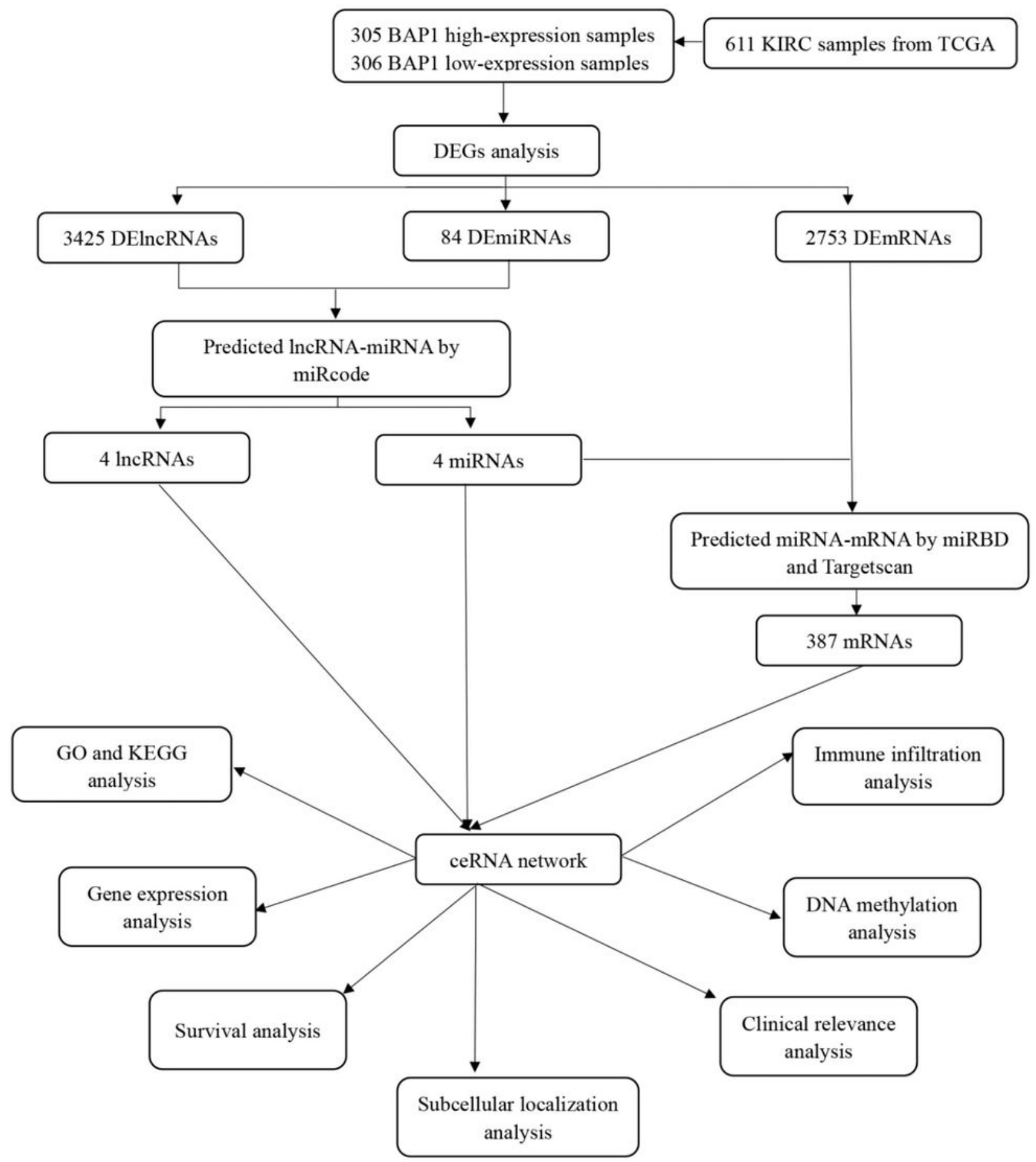

\section{Figure 1}

Flow diagramm of ceRNA construction and analysis. 
A

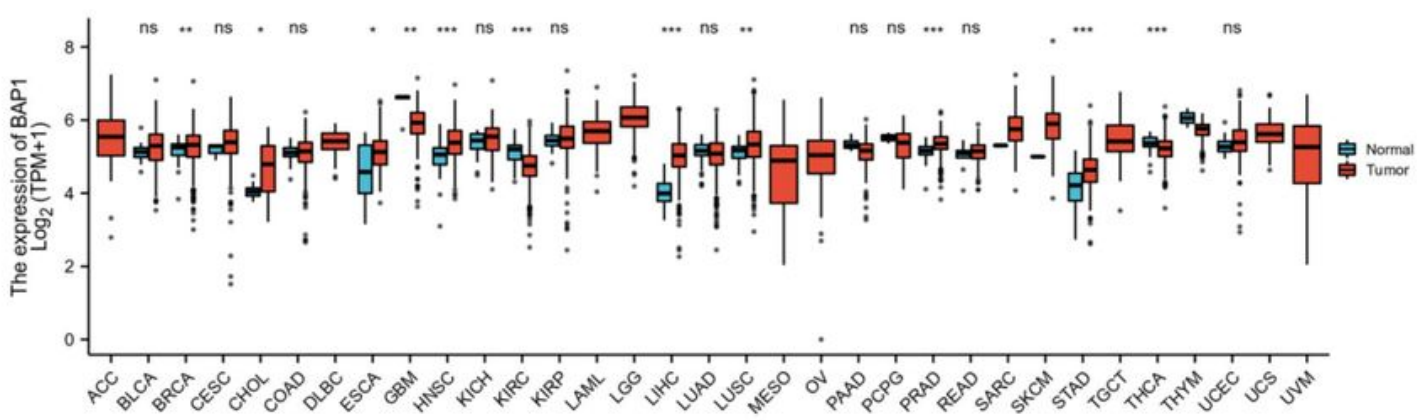

B

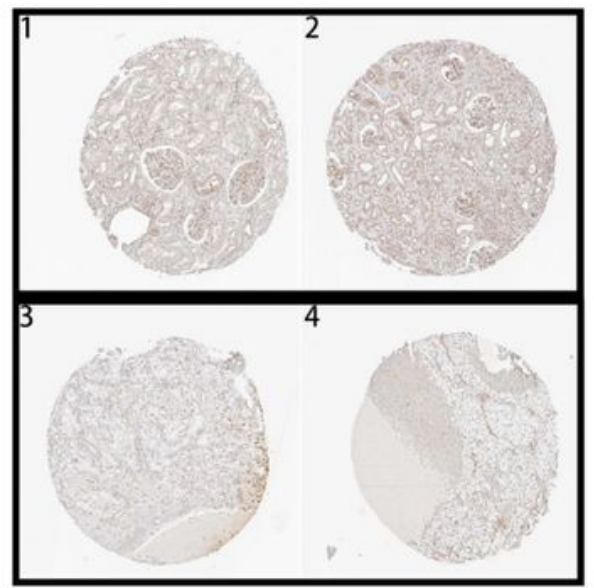

Normal Tissue C

\section{Tumor Tissue}

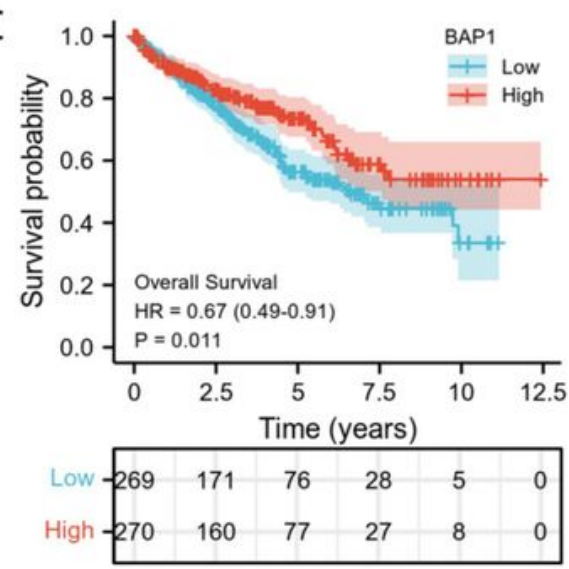

D вмค

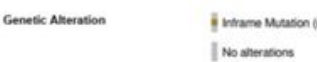

E

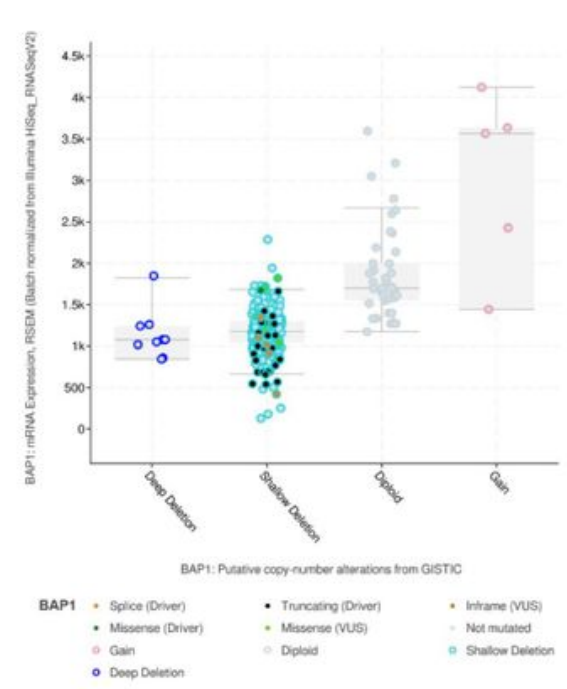

$\mathrm{F}$

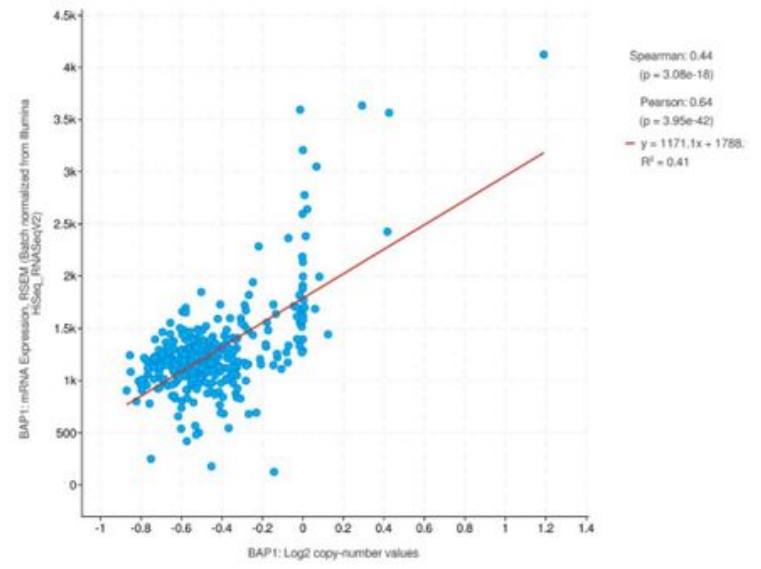

Figure 2

BAP1 acts as a tumor suppressor in kidney renal clear cell carcinoma (KIRC). (A) Pan-cancer analysis of BAP1; (B) Immunohistochemical analysis of BAP1 in renal tumor and normal tissues; (C) Survival analysis comparing high- and low expression of BAP1; (D) Distribution of BAP1 genomic alterations inTCGA KIRC; (E, F) Relationship between copy number alterations and BAP1 expression: scatter plot (E), correlation plot $(\mathrm{F})$. 

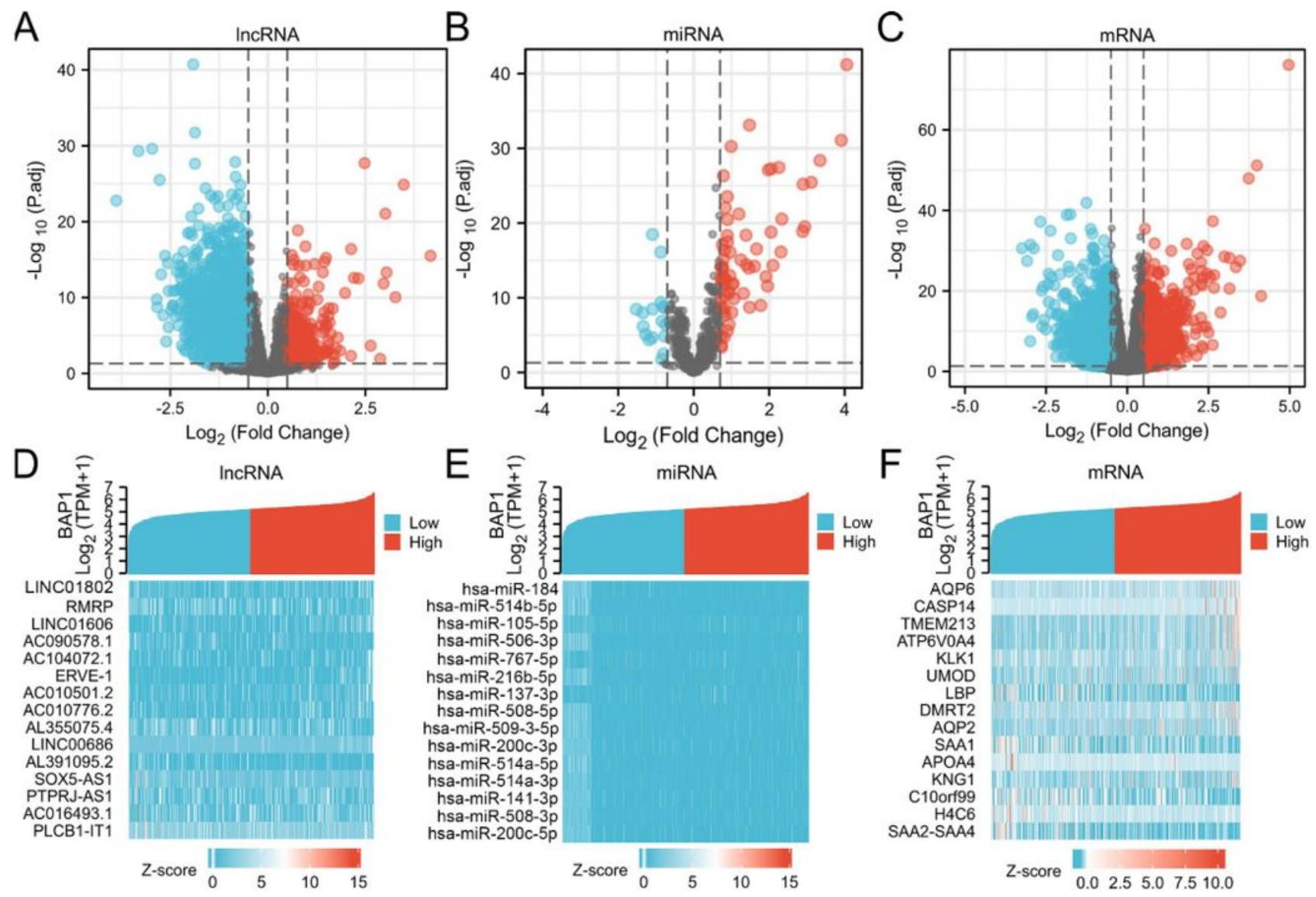

\section{Figure 3}

Volcano plots and heatmap plots of DElncRNAs, DEmiRNAs, and DEmRNAs between the expression of BAP1high and BAP1low in KIRC samples. Red color represents up-regulated genes, blue represents downregulated genes. (A) 3425 DElncRNAs ( $|\log 2 \mathrm{FC}|>0.5$ and P. adj < 0.05); (B) 84 DEmiRNAs ( $|\log 2 \mathrm{FC}|>0.7$ and P. adj < 0.05); (C) 2753 DEmRNAs with cutoff value of $\| \log 2 \mathrm{FCl}>0.5$ and $\mathrm{P}$. adj < 0.05; (D-F) Heatmaps of the top 15 significant DElncRNAs, miRNAs and mRNAs. 
A

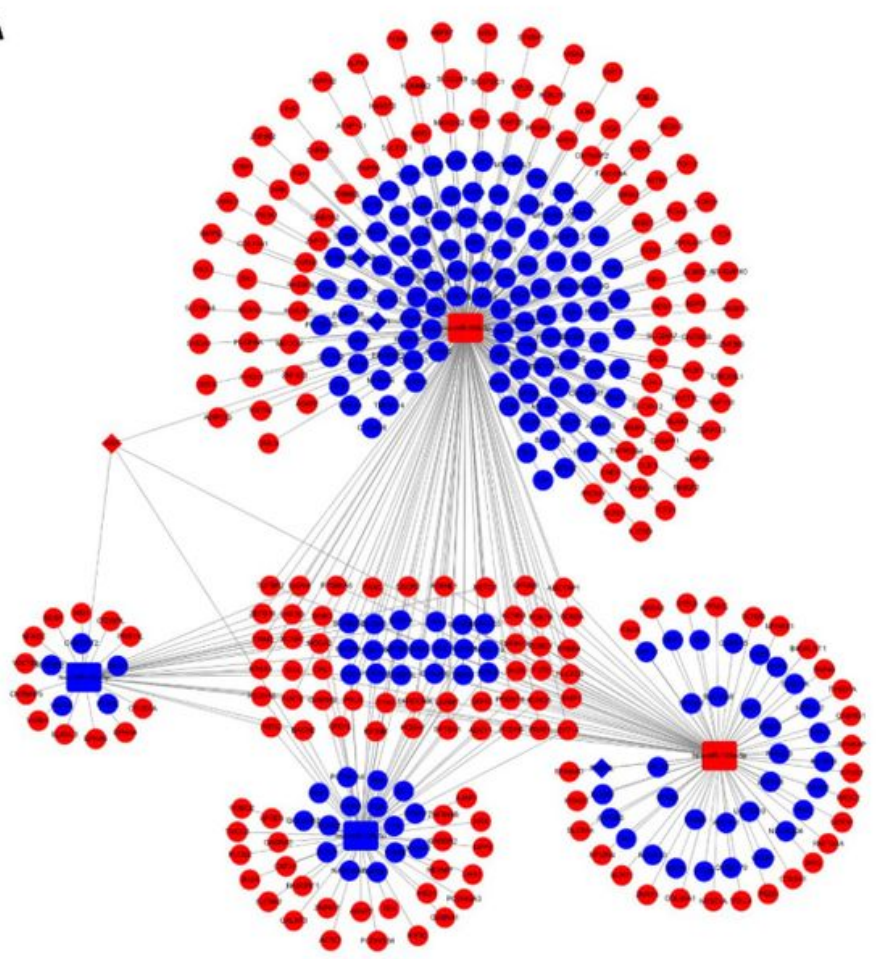

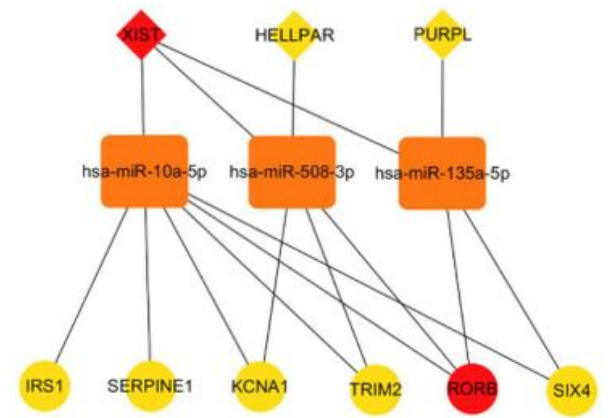

C

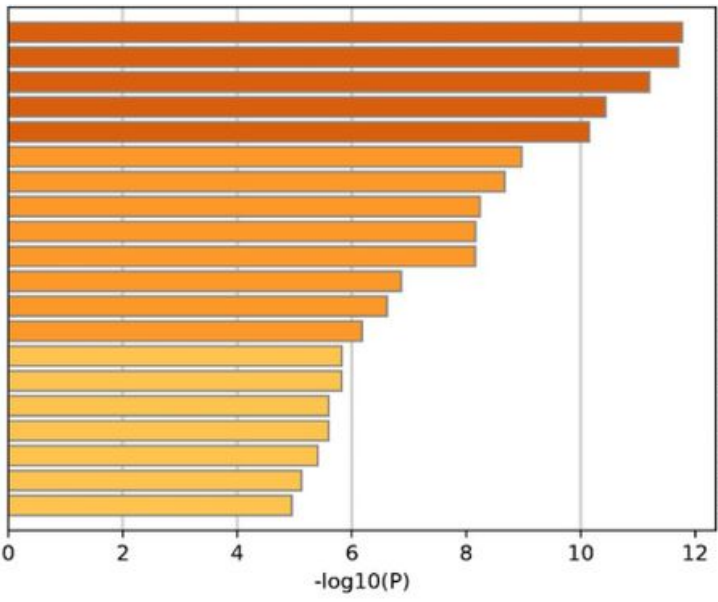

GO:0045596: negative regulation of cell differentiation GO:0007423: sensory organ development GO:0021953: central nervous system neuron differentiation GO:0001944: vasculature development GO:0099537: trans-synaptic signaling GO:0003281: ventricular septum development GO:0061061: muscle structure development GO:0045165: cell fate commitment GO:0070848: response to growth factor GO:0007416: synapse assembly

GO:0001655: urogenital system development

GO:0060485: mesenchyme development

GO:0048589: developmental growth

GO:0048665: neuron fate specification

GO:0060541: respiratory system development

R-HSA-112316: Neuronal System

GO:0030155: regulation of cell adhesion

GO:0007389: pattern specification process

WP4331: Neovascularisation processes

GO:0007169: transmembrane receptor protein tyrosine kinase signaling pathway

\section{Figure 4}

Construction of ceRNA networks and functional annotation. (A) A triple regulatory network based on 4 IncRNAs, 4 miRNAs, and 387 mRNAs; (B) Hub genes network was constructed using plug-in "cytoHubba"; (C) Functional enrichment analysis of DEmRNAs. 

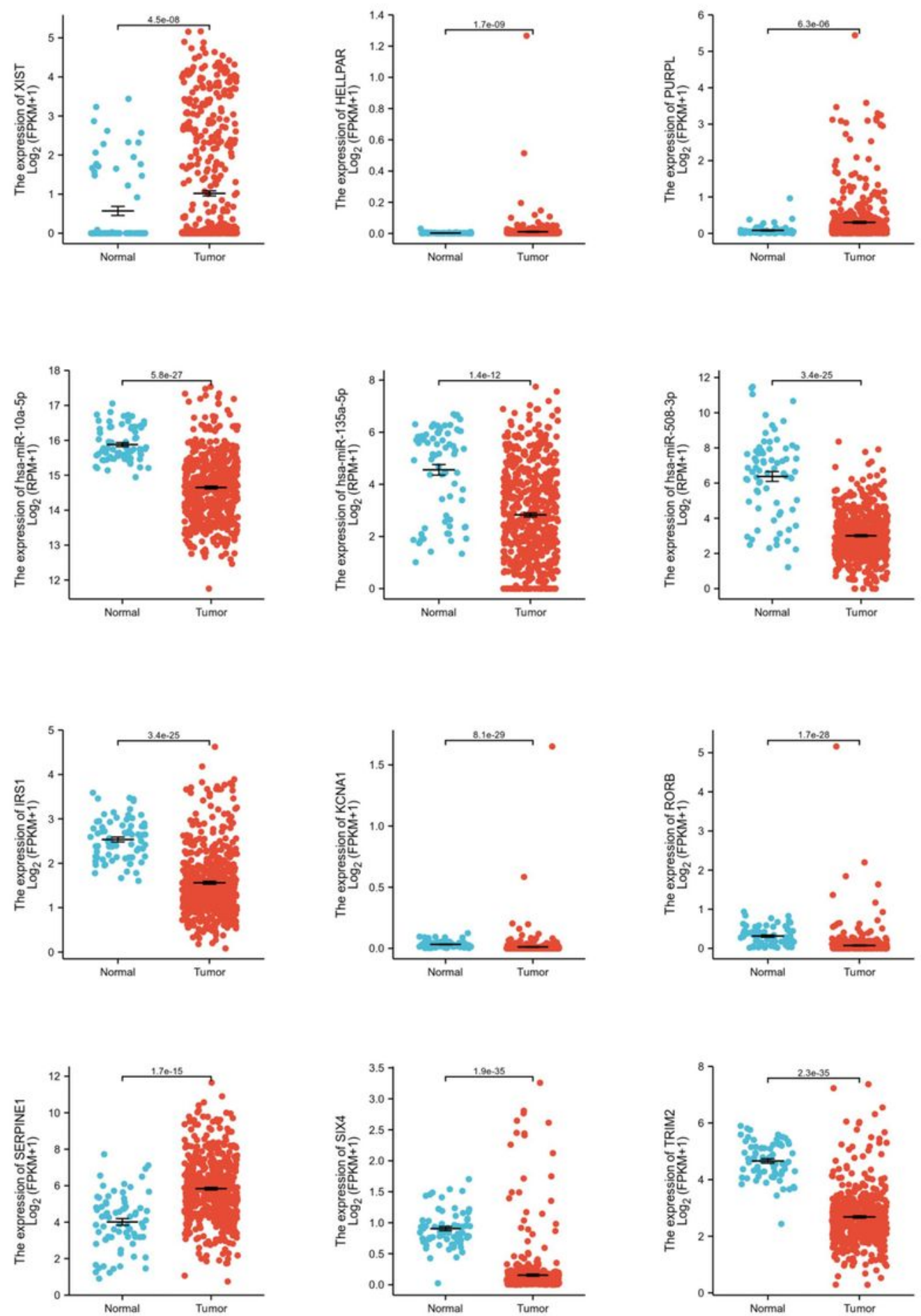

Figure 5

Hub genes expression analysis. Expression analysis of 12 hub genes ( 3 IncRNAs, 3 miRNAs, 6 mRNAs) comparing tumor and normal tissues. 

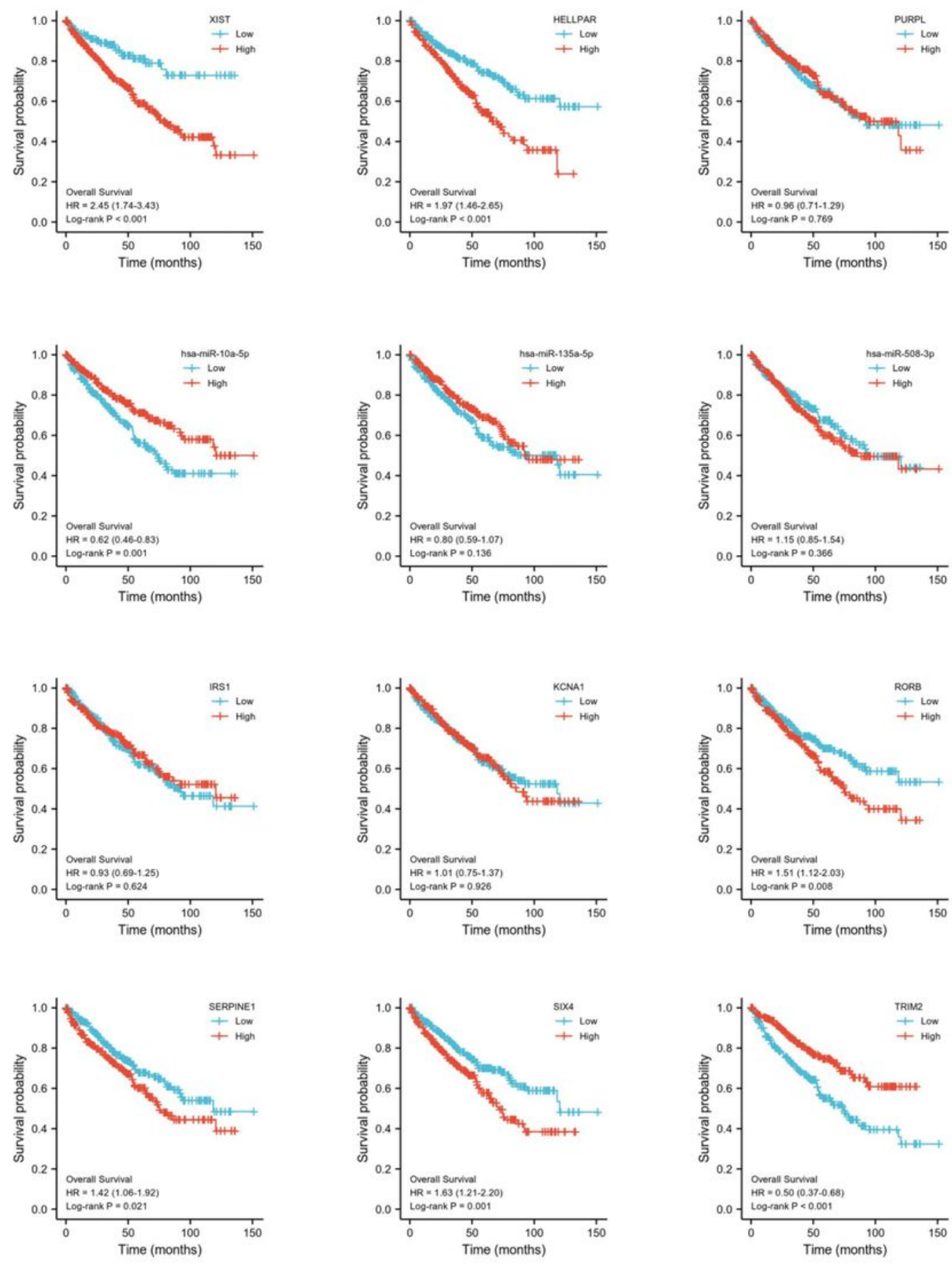

\section{Figure 6}

Hub genes survival analysis. Survival analysis of 12 hub genes (3 IncRNAs, 3 miRNAs, 6 mRNAs) comparing high- and low expression group. 


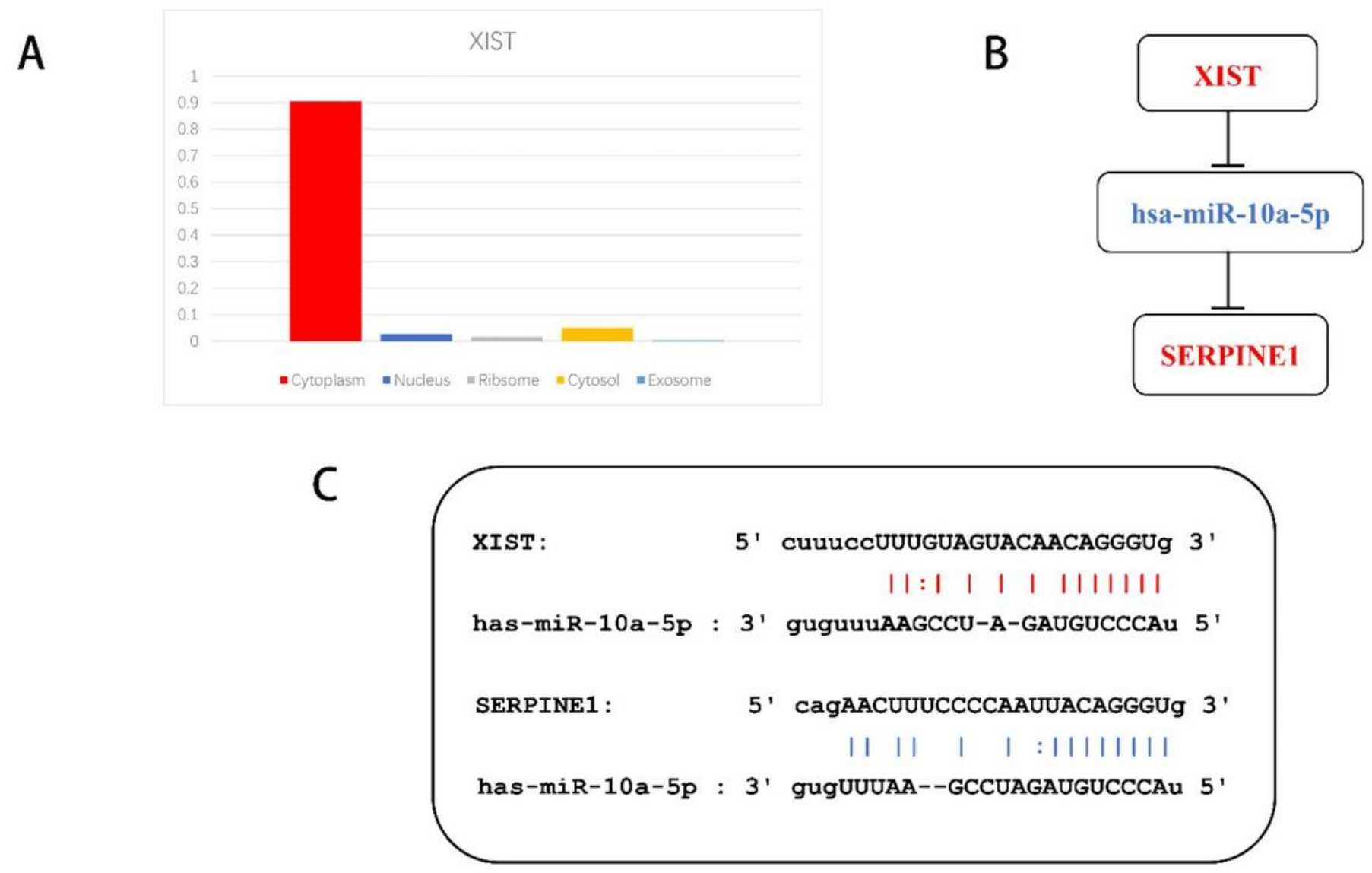

Figure 7

Construction of ceRNA. (A) XIST was found to be located in cytoplasm through subcellular localization analysis; (B) Regulation model of XIST/miR-10a-5p/SERPINE1 axis, red indicated up-regulated, blue indicated down-regulated; (C) Predicted IncRNA-miRNA, miRNA-mRNA binding sites by Targetscan. 
A
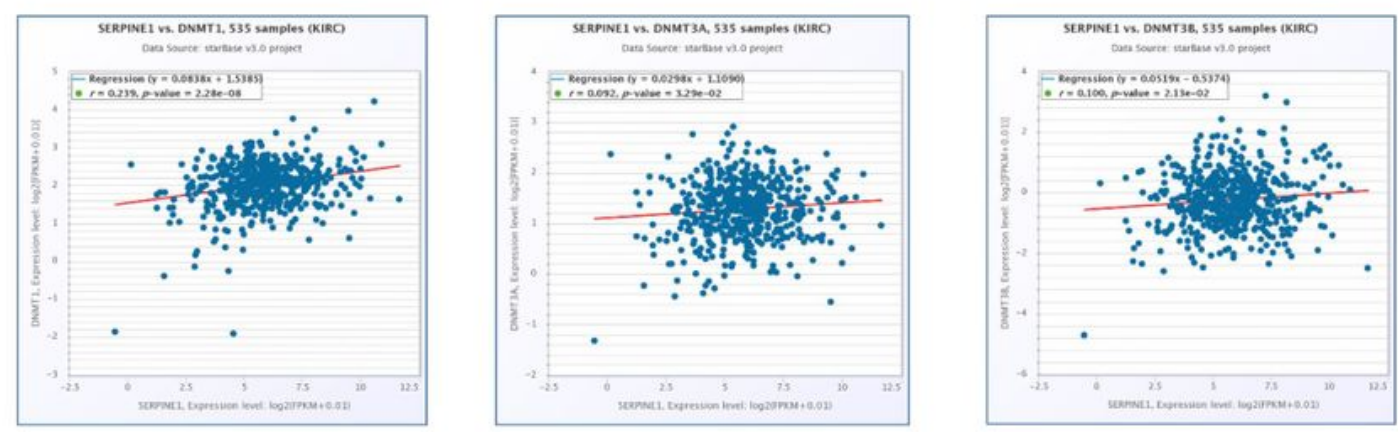

B

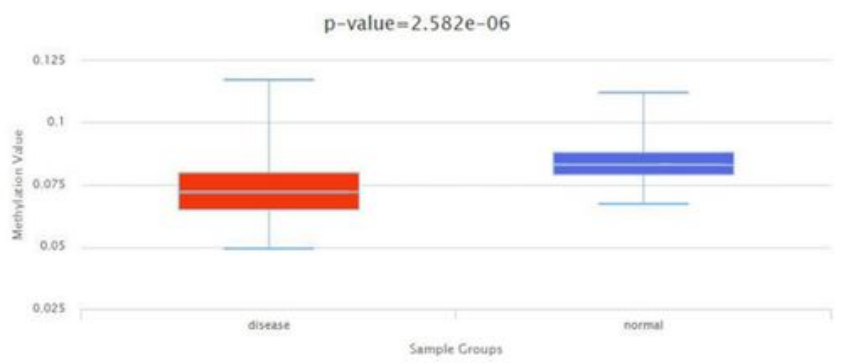

c
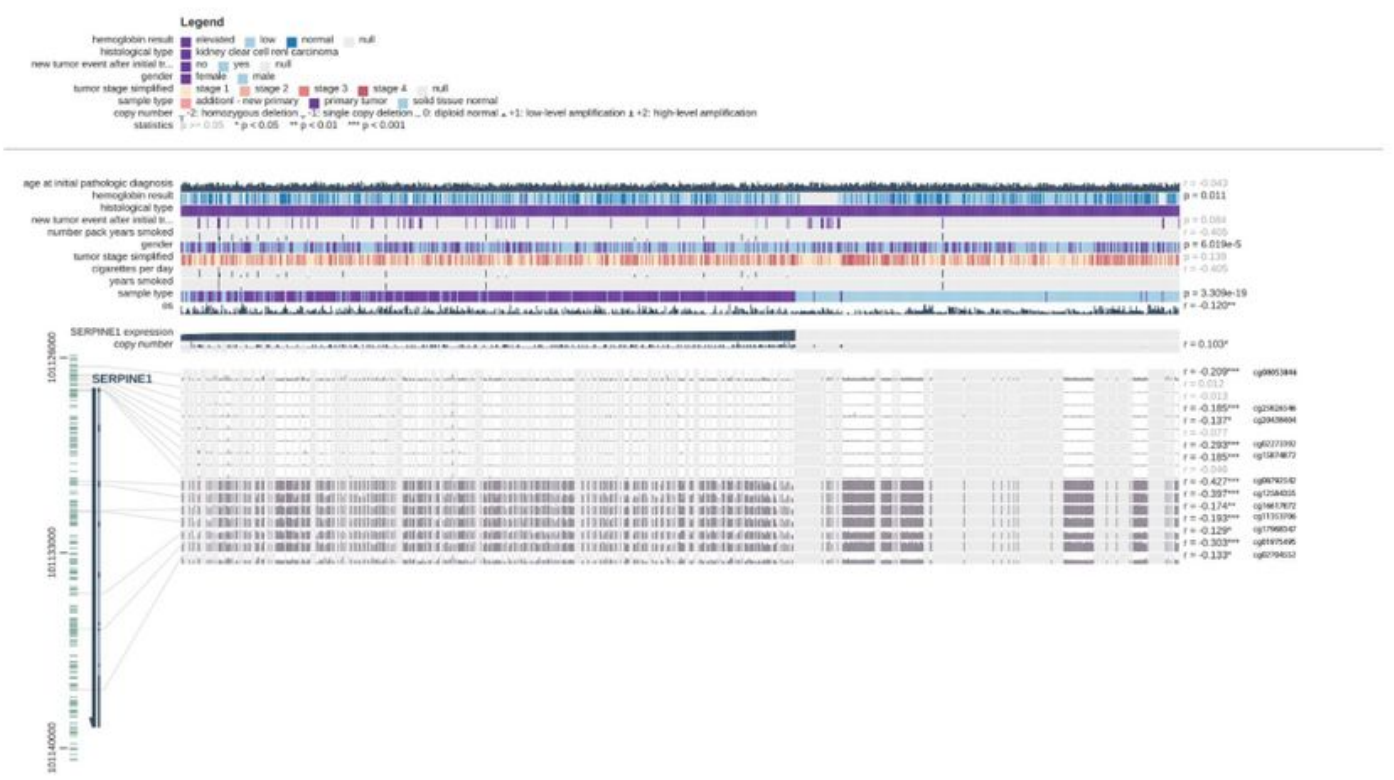

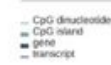

\section{Figure 8}

DNA methylation analysis of SERPINE1. (A) Co-expression analysis of SERPINE1 with three DNMTS (DNMT1, DNMT3A and DNMT3B); (B) Methylation level of SERPINE1 comparing KIRC and normal samples; (C) Relationship between SERPINE1 expression and genome-wide methylation by MEXPRESS. 
A

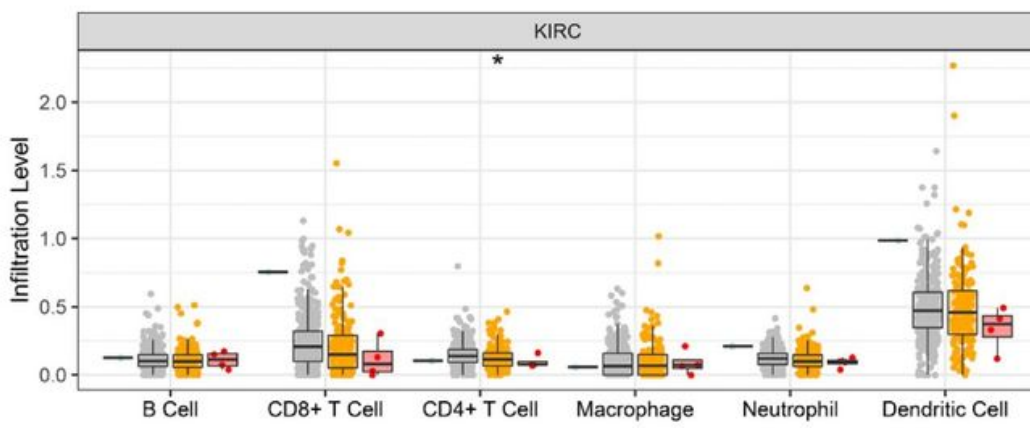

Copy Number

由 Arm-level Deletion

审 Diploid/Normal

审 Arm-level Gain

审 High Amplication

B

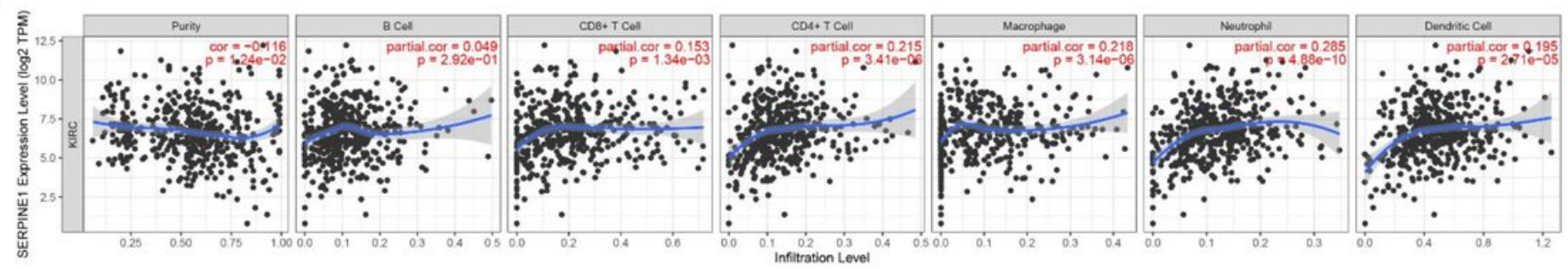

Figure 9

Immune infiltration analysis of SERPINE1 in KIRC. (A) Relationship between the level of immune cell infiltration and the copy number of SERPINE1 in KIRC. (B) Correlation of SERPINE1 expression and immune cell infiltration in KIRC. ${ }^{\star} p<0.05,{ }^{*} \mathrm{p}<0.01,{ }^{* \star} \mathrm{p}<0.001$.

\section{Supplementary Files}

This is a list of supplementary files associated with this preprint. Click to download.

- supplementary.docx 